\title{
Ontology of homologous series
}

\author{
V. Suslov \\ Institute of Cytology and Genetics SB RAS, Novosibirsk, Russia \\ e-mail:valya@bionet.nsc.ru
}

Key words: parallel evolution, Vavilov's law of homologous series

Motivation and Aim: It is assumed that the Vavilov's law of HS must unite all events of parallelisms, but the description of the intrinsic trends of autoadaptation and stabilization of homologous series (HS) raised the question of the types of HS.

Results: We can talk about the bouquet of HS laws. In the literature described HS for inherited traits of adult forms living syn- (Vavilov) and diachronously (Sobolev), for successions of transformational trends of these traits (Cope's homo- and heterologous series) and structural completeness for these trends (Kammerer's Gesetz der Serie and Meyen's refrains); HS for norm and pathology (Krenke, Rostand), between pathologies (Rostand), norm and stress (Suslov) and norm of reaction in norm and under the stress (Gorban) and between norms (Walsh); HS for sexual dimorphism (Efremov and Geodakjan's rules); HS for inherited expression/location of the traits in body space (Ugolev's universal blocks) and the same for inherited and non-inhereted expression/ location (Iordansky) and finally HS for onto- and phylogenesis (Mueller-Haeckel and von Baer laws), for ontogenesies (von Baer laws) for inherited traits and noninherited modification (Lamarck) and HS between living and nonliving nature (Meyen's refrains). Four concepts of these HS explicated. 1) Vavilov's idea. HS evolution reduced to the evolution of traits in a limited space of possibilities ${ }^{1}$. 2) Kammerer's idea. HS is a consequence of the regulation of several subsystems by a supersystem: a priori ${ }^{2}$, permanently ${ }^{3}$ or not $-\mathrm{HS}$ can exist by inertia ${ }^{4}$. Meyen's refrain can be found in all three subtypes. 3) The HS as program (transposition etc.). 4) The HS as result of autoadaptation. This HS-4 is logically incompatible with the HS-2, but not HS-1 and 3 and can explain the incorrectness in them.

Acknowledgements: Supported by the grant 0324-2018-0017 and IP 0324-2018-0021.

\footnotetext{
${ }^{1}$ For example, classic Vavilov's HS based on the geographical space of centers of diversity and Zavarzin HS based on the Zavarzin-Sax's space of logical possibilities.

${ }^{2}$ Periodic Mendeleev system and other correlative nonliving, suborganismal and superorganismal systems functioning on the Maupertuis principle, but not linearly additive as HS-4.

${ }^{3}$ For example, Belyaev's HS of domesticated forms usually accepted as Vavilov's HS. In sensu stricto this is not so. Vavilov's HS includes both domesticated and wild forms and originates within centers of diversity that characterized by an abundance of rare dominant alleles. Belyaev's HS revealed only between domesticated forms: traits revealed during the domestication of foxes are parallel to those of domesticated mink, horse, cattle and, finally, deer but not found in any wild species of Carnivora and ungulates. De novo traits in Belyaev's HS revealed as semi-dominant but not dominant. Belyaev's HS is more logical to compare with the Rostand and Krenke HS, if we consider domestication as a pathology.

${ }^{4}$ Most purely represented in mechanics. Unsuccessfully searched by Kammerer and Lyubishchev (as style phenomenon) in biology among the inherited traits of the archetype. However, were successfully found in biology by Gorban (the non-inherited effect of group stress) and Efremov and Geodakjan (inherited traits of sexual dimorphism without archetype).
} 